\title{
High-Frequency Orographically Forced Variability in a Single-Layer Model of the Martian Atmosphere
}

\author{
CHRISTIAN L. KEPPENNE \\ Jet Propulsion Laboratory, California Institute of Technology, Pasadena, California \\ ANDREW P. INGERSOLL \\ Division of Geological and Planetary Sciences, California Institute of Technology, Pasadena, California
}

(Manuscript received 25 August 1993, in final form 7 November 1994)

\begin{abstract}
A shallow water model with realistic topography and idealized zonal wind forcing is used to investigate orographically forced modes in the Martian atmosphere. Locally, the model produces barotropic modes with periods within the broad range of periods observed at the sites of Viking Lander I and II (VL1 and VL2) during the fall and spring seasons. Its variability at those sites is dominated by an oscillation of 3 Martian solar days (sols) in the region of VL1 and by a 6-sol oscillation in that of VL2. These oscillations are forced by the zonal asymmetries of the Martian mountain field. Their robustness with respect to changes of the fundamental model parameters is examined. Since the exhibited periods occur for a barotropic forcing field that is highly idealized, it is difficult to say whether they have much to do with the real Mars, but their resemblance to some of the periodicities present in the observed Martian climatology deserves further investigation.

The spatial variability associated with the orographically forced oscillations is studied by means of extended empirical orthogonal function (EEOF) analysis. The 3-sol VL1 oscillation corresponds to a tropical, eastward traveling, zonal wavenumber one pattern. The 6-sol VL2 oscillation is characterized by two midlatitude, eastward traveling, mixed zonal wavenumber one and two and zonal wavenumber three and four patterns, with respective periods near 6.1 and 5.5 sols. The corresponding phase speeds are in agreement with some of the conclusions drawn from the lander observations. A linear stability analysis of the zonally asymmetric climatology reveals that the two most unstable modes are associated with periods near 3 and 6 sols; with the corresponding eigenvectors showing patterns consistent with the results of the EEOF analyses.
\end{abstract}

\section{Introduction}

In pioneering studies of the Martian atmosphere (Mintz 1961; Leovy 1969), baroclinic instability was recognized as the primary factor leading to the eastward-traveling waves that characterize the Martian atmosphere's synoptic variability. After the observations made by the Mariner 9 spacecraft, the Viking Lander 1 and 2 (VL1 and VL2) spacecrafts revealed disturbances with periods in the range of 2-10 Martian solar days (sols). The lander observations were analyzed by Barnes $(1980,1981)$. They exhibit a broad range of spectral peaks dominated by substantial and consistent variance in the period range 2-3 sols thoughout all the data records at both sites. In the spring and fall seasons, the VL1 pressure observations also show almost regular oscillations with periods near 3.0 and 3.7 sols, respectively. Although they are

Corresponding author address: Dr. Christian L. Keppenne, Mail Stop 238-332, Jet Propulsion Laboratory, 4800 Oak Grove Dr., Pasadena, CA 91100. not generally dominant, periodicities near 6.7 and 8.0 sols occur at VL2 during the same seasons. It is shown in the remainder of this paper that similar periodicities can occur in a barotropic shallow water model with idealized equinoctial zonal forcing where they can be attributed to linear instabilities of the topographically forced barotropic flow.

With the help of the quasigeostrophic and baroclinic instability (Charney 1947; Eady 1949) theories, Barnes $(1980,1981)$ hypothesized the baroclinic character of the observed disturbances and determined the approximate zonal wavenumbers associated with them. In a later paper, Barnes ( 1984) used quasigeostrophic linear baroclinic models with and without a zonally symmetric topography to examine baroclinic instability in the Martian atmosphere. Zonal wavenumber three and four disturbances were found to be the most unstable in the midlatitudes, in agreement with the conclusions inferred from the lander data (Barnes 1980, 1981). In the model with topography, the zonally symmetric mountain field was found to have a substantial stabilizing effect.

In the light of the above results and of observations from related studies (e.g., Leovy 1979; Pollack et al. 
1981, Barnes et al. 1993; Haberle et al. 1993), the importance of baroclinic instability in the generation of midlatitude synoptic waves is now widely recognized. In the present paper, we show that barotropic orographically forced waves induced by the interaction of an idealized zonal flow with the zonally asymmetric component of the Martian mountain field in a shallow water model can also occur at periodicities within the broad range of observed synoptic variability. The importance played by regional topography in Martian atmospheric variability was first suggested by the observational study of Murphy et al. (1990).

This study is motivated by the findings of a series of papers (Legras and Ghil 1985; Ghil 1987; Jin and Ghil 1990; Marcus et al. 1990; Tribbia and Ghil 1990; Strong et al. 1993; Keppenne and Ghil 1995) in which the role played by orographically induced disturbances in the intraseasonal variability of Earth's atmosphere was elucidated. Since the ratios of the irregularities of Earth's and Mars' orographies to the scale heights of their respective atmospheres are within the same order of magnitude, it is likely that orographic oscillations such as those observed on Earth will also play a role in Martian meteorology. Nevertheless, this ratio is still several times higher on Mars than it is on Earth. Thus, one should not be surprised to find differences in the nature of the meteorological responses to these planets' mountain fields.

We use a spherical shallow water spectral model with realistic topography. Linear drag tends toward a steady zonal. flow typical of equinox conditions. This idealized forcing allows us to study the role of the Martian mountain field in the generation of barotropic waves. At this point, it is necessary to introduce the caveat that, aside from the basic problem that the Martian atmosphere is highly baroclinic, thereby making it difficult to choose an appropriate barotropic forcing flow for this model, most of the Viking data analyses were performed for periods where the zonal flow is different from that near equinox. The equinoctial forcing flow is only relevant for near-equinox conditions (Haberle et al. 1993).

The model's single-layer configuration precludes baroclinic instability - a mechanism by which zonal mean potential energy is converted to eddy potential energy in a stratified fluid-from manifesting itself. Moreover, barotropic instability - that is, the conversion of zonal mean kinetic energy to eddy kinetic energy in the presence of an appropriate potential vorticity gradient--is also disallowed in the absence of the zonal asymmetries of the mountain field, because the zonal forcing is chosen to be barotropically stable when those asymmetries are ignored. The model's departure from a steady state is due to the longitudinal variability of the Martian mountain field. If this variability is removed but the zonally averaged topography is retained, the model evolves toward an asymptotic steady state characterized by the relaxation of the streamfunction field to the zonal forcing.

In a previous paper (Keppenne 1992) it was shown that the Martian mountains induce low-frequency global oscillations with periods near 85 and 125 days in the model. In the present study, we switch our attention to the range of shorter periodicities that are the most manifest in the lander observations.

The remainder of the paper is organized as follows. The model is presented briefly in the next section, followed by a spectral analysis of model-generated time series at the sites of VL1 and VL2. The robustness of the variability present in those time series is examined by changing the topographic height and the Newtonian cooling. The zonal structure of the variability at the lander sites is then examined by means of extended empirical orthogonal functions (EEOF) analysis. Section 4 concludes with a discussion of the results.

\section{The model}

We use the spectral shallow water model detailed in Keppenne (1992). The dimensionless system of prognostic equations is

$$
\begin{aligned}
& \partial_{t} \Delta \psi=-\nabla \cdot {[\mathbf{u}(\Delta \psi+f)]-\tau U \Delta \psi } \\
&+(-)^{n+1} \gamma \Delta^{n} \psi-\epsilon \Delta\left(\psi-\psi^{*}\right), \\
& \partial_{t} \Delta \chi=\mathbf{k} \cdot \nabla \times[\mathbf{u}(\Delta \psi+f)]-\tau U \Delta \chi \\
&+(-)^{n+1} \gamma \Delta^{n} \chi-\Delta\left[\frac{\mathbf{u} \cdot \mathbf{u}}{2}+\phi\right], \\
& \partial_{t} \phi=-\nabla \cdot\left[\mathbf{u}\left(\phi-\phi_{b}\right)\right]-\left(\varphi-\varphi_{b}\right) \Delta \chi
\end{aligned}
$$

where $(1 a-b)$ is the vorticity-divergence form of the momentum equation and (1c) is the mass-conservation equation. Here, $\psi(\lambda, \theta, t), \chi(\lambda, \theta, t)$, and $\phi(\lambda, \theta, t)$, are the streamfunction, velocity potential, and the deviation of the geopotential height of the free surface $h(\lambda, \theta, t)$, representing the top of the atmosphere, from its global average $\varphi$, so that $\phi+\varphi=g h$, where $g$ is the acceleration of gravity. Here $\lambda$ is longitude, $\theta$ latitude, and $t$ time. The dimensionless time unit is equal to the Martian rotation period $(2 \pi / \Omega=1.0259$ day $)$, and the dimensionless length unit is $R$, the equatorial radius of Mars $(3483 \mathrm{~km})$. Here $\mathbf{u}(\lambda, \theta, t)$ is the horizontal fluid velocity, $f=2 \Omega \sin \theta$ is the Coriolis parameter, $\tau$ is a drag coefficient, $\gamma$ is a diffusion coefficient, $U$ is the instantaneous global-mean square-root zonal kinetic energy per unit mass, $\phi_{b}(\lambda, \theta)$ is the topography, and $\Delta$ is the Laplacian operator.

The relaxation term, $\epsilon \Delta\left(\psi-\psi^{*}\right)$, is applied only to the spectral coefficients corresponding to zonal spherical harmonics. The forcing $\psi^{*}(\theta)$ corresponds to a forcing wind field, $u^{*}(\theta)=-\partial \psi^{*}(\theta) / \partial \theta$, which has 


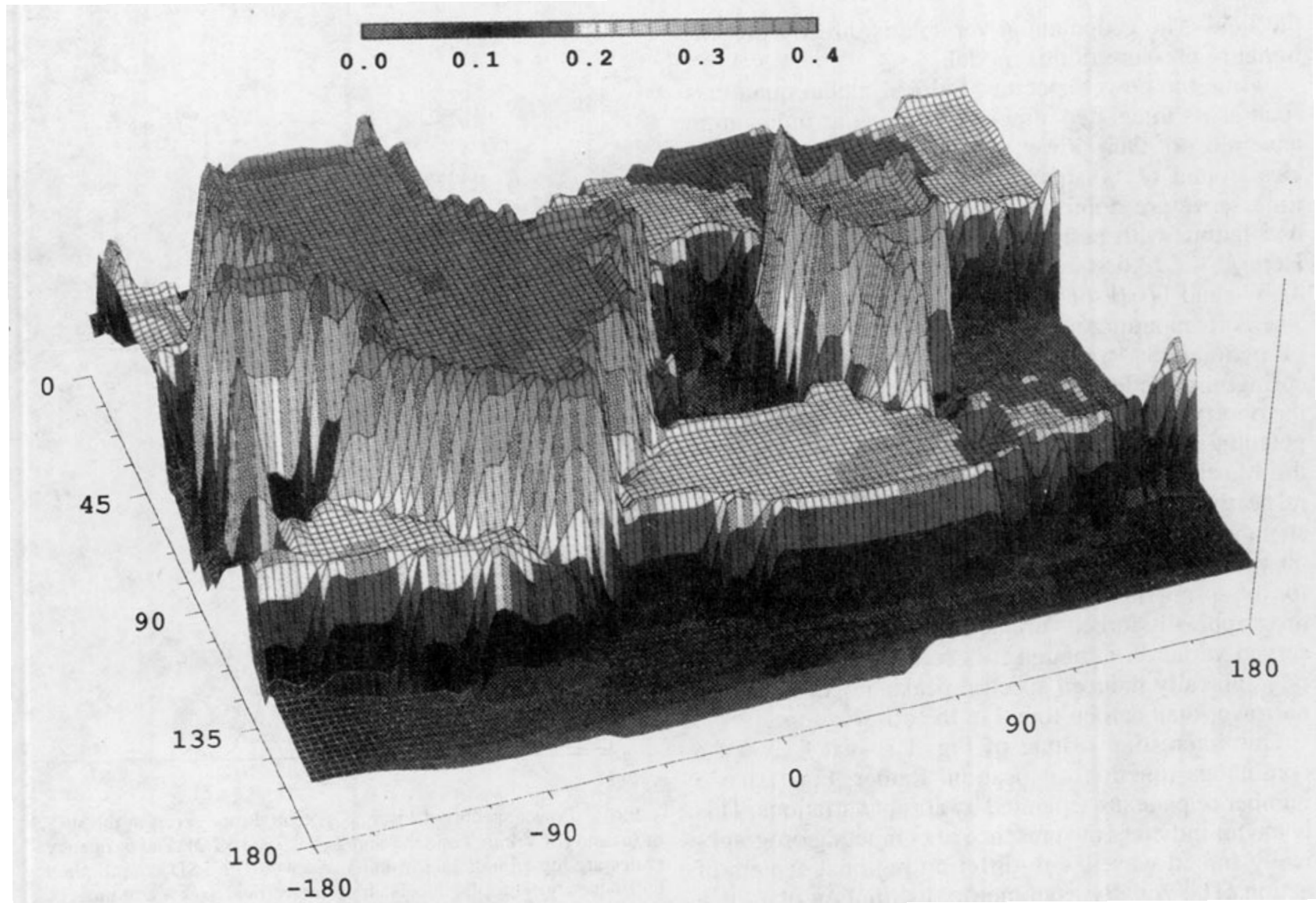

FIG. 1. Geographical distribution of $f m$, the frequency associated with the highest power spectral density (PSD) of the model's geopotential field. The horizontal axes show the colatitude and longitude in degrees and $f m$ is expressed in cycles sol ${ }^{-1}$.

a midlatitude jet of $30 \mathrm{~m} \mathrm{~s}^{-1}$ and is symmetric about the equator to account for the effect of equinoctial solar heating on the Martian circulation. As mentioned in the introduction, this idealized forcing field is only an appropriate barotropic representation of the highly baroclinic Martian flow for near-equinox conditions. When the longitudinal variability of the mountain field is ignored, the forcing field and the model's response to it satisfy the criterion for barotropic stability (e.g., Pedlosky 1987): the poleward gradient of the absolute vorticity, $\Delta \psi+f$, does not change sign anywhere between the poles. However, the model's response to $\psi^{*}$ becomes unsteady when the longitudinal variations of the Martian orography are accounted for, leading to the variability discussed in the following sections.

The free surface of the shallow water layer is representative of the 2 -mb pressure level $\left[\varphi-\varphi_{b}=30\right.$ $=g h(2 \pi / \Omega)^{2} / R^{2}, h=30 R^{2} \mathrm{sol}^{-2} / g \approx 13 \mathrm{~km}$ in (1c)]. Here $\tau$ is 0.1 and the drag terms in $(1 \mathrm{a}-\mathrm{b})$ are applied selectively to the spectral coefficients corresponding to nonzonal spherical harmonics. The relaxation time, $1 / \epsilon$, is 10 sols; $n=4$ to selectively damp high-wavenumber oscillations; and $\gamma$, the diffusion coefficient, is $5 \times 10^{-12}$. As a result, a wavenumber-20 disturbance will damp with a time constant of approximately 6 days.

The spectral form of (1) is integrated at rhomboidal truncation R20 using an associated Gaussian grid with 50 Gaussian latitudes and 64 meridians and a realistic mountain field interpolated from the topography of the NASA Ames Martian GCM (e.g., Pollack et al. 1990). This topography is shown in Fig. 8a. The transform method (Orszag 1970; Eliassen et al. 1970; Bourke 1972 ) is used to iterate back and forth between the gridpoint and spectral representations of the model variables.

\section{Results}

The model was run for 6000 sols from the initial condition of an atmosphere at rest $(\psi=\chi=\phi=0)$, and the first 1000 sols of model history were discarded to eliminate undesired transients. The model history was sampled once daily at every gridpoint. Figure 1 gives a global picture of the variability during the last 5000 sols of the run by showing the spatial distribution of $f_{m}(\lambda, \theta)$, the frequency associated locally with the highest power spectral density (PSD) of the geopoten- 
tial field. The geopotential variable is directly proportional to pressure in this model.

While the power spectra of global model quantities such as its integrated atmospheric angular momentum have most of their power associated with low frequencies around 1/100 sols $^{-1}$ (Keppenne 1992), the local time series are dominated by much higher frequency oscillations with periods in the range of 2.5-10 sols. Here $f_{m}$ is $1 / 3.6 \mathrm{sol}^{-1}$ at the location of VL1 $\left(22^{\circ} \mathrm{N}\right.$, $\left.48^{\circ} \mathrm{W}\right)$ and $1 / 6.0 \mathrm{sol}^{-1}$ at the VL2 site $\left(48^{\circ} \mathrm{N}, 134^{\circ} \mathrm{E}\right)$. The corresponding periods are within the broad range of periodicities found between 2 and 8 sols in the springtime lander observations. Also consistent with the observations are the ratios $\sigma_{\phi} /\left(\varphi-\varphi_{b}\right)$ of the geopotential standard deviation to the modeled depth of the Martian atmosphere. These are 0.009 and 0.015 , respectively, at the sites of VL1 and VL2. The corresponding ratios are 0.008 and 0.016 for the pressure observations in the fall season, and 0.0065 and 0.019 for the spring observations. In other words, the model's orographically forced variance is comparable to the observed variance, although it is seen below that the topographically induced spectral peaks are stronger and narrower than can be found in the observations.

One interesting feature of Fig. 1 is that $f_{m}$ is not a continuous function of location. Rather, Fig. 1 has a number of plateaus separated by abrupt variations. This behavior indicates the presence of competing orographically forced waves with differing regional centers of action. The zonally asymmetric distribution of $f_{m}$ is a sign that the corresponding variability is induced by the mountain field. Indeed, if it were not for the zonally asymmetric topography, the time-independent model statistics would have a zonal distribution reflecting the zonal symmetry of the forcing.

Figure 2 shows power spectra of the geopotential variable at the sites of VL1 and VL2. These power spectra are computed by the maximum entropy method (MEM: Yule 1927; Walker 1931; Burg 1968) using Burg's (1968) algorithm. Sixty poles are used in the computations. The geopotential power spectrum at VL1 (Fig. 2a) shows a pronounced peak centered at a frequency of $0.28 \mathrm{sols}^{-1}$. The corresponding period of 3.6 sols is close to a 3.7-sol period found in the VL1 fall pressure observations (Barnes 1980), and not too distant from the 3.0 -sol period, which is a pronounced feature of the spring observations at the same site. The model variability in the region of VL2 is dominated by a 6.0-sol oscillation whose signature is the most striking feature of Fig. 2b. Although the observations generally show a wide range of broad spectral peaks at both lander sites, this result is also consistent with some of the VL2 pressure observations: Barnes (1980) reports a very distinct peak corresponding to a period of 6.7 sols in the power spectrum of the VL2 pressure during the fall season, and another with an associated period of 8 sols in the springtime observations at the same location. (a)

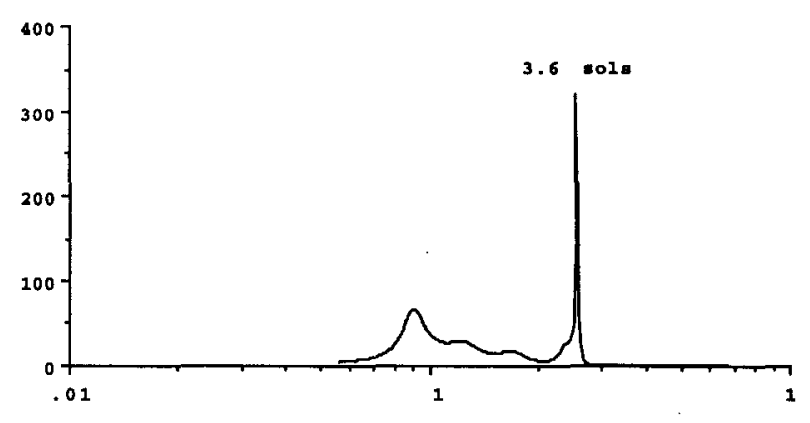

(b)

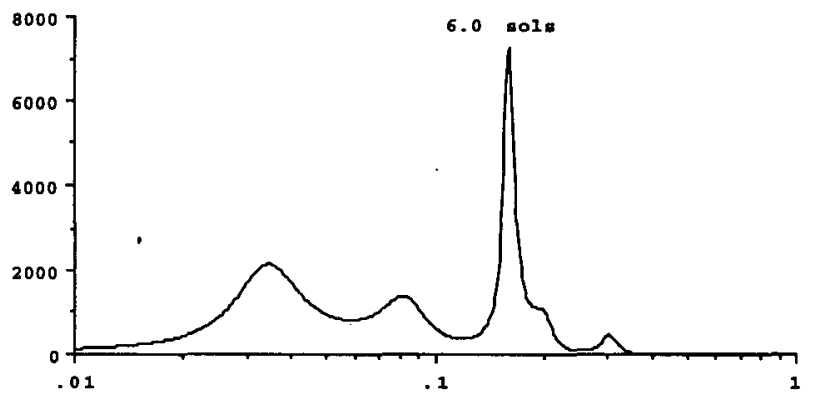

Fig. 2. Power spectra of local geopotential time series at the sites of (a) and (b) Viking Lander 1 and 2 (VL1 and VL2). The frequency scales are logarithmic and in units of sol $^{-1}$. The PSD is multiplied by frequency. Thus, the labels along the vertical axes are in units of $\mathrm{m}^{2}$.

To determine how robust the model variability is with respect to changes of the model parameters, we show in Fig. 3 MEM power spectra of the model's total atmospheric angular momentum (AAM) for a number of 1000-sol integrations with different settings, after discarding undesired transients. In all these power spectra, the integral of the PSD between the bounds of $0.01 \mathrm{cycles} \mathrm{sol}^{-1}$ and $1.0 \mathrm{cycles} \mathrm{sol}^{-1}$ is normalized to unity.

Figures $3 a-f$ each correspond to rescaling the mountain field by multiplying it by a constant number $\mu$ between 0.0 and 1.5 . Figure $3 \mathrm{~d}$ corresponds to the nominal settings $(\mu=1.0)$. The integration leading to Fig. 3a has no topography at all $(\mu=0.0)$, and $\mu$ is 0.5 , $0.75,1.25$, and 1.5 in the model runs corresponding to Figs. 3b, 3c, 3e, and 3f, respectively. Generally speaking, the AAM variability is characterized by lower-frequency oscillations than those encountered in the local geopotential power spectra of Fig. 2 (Keppenne 1992). A split peak corresponding to periodicities near 3 sols is nevertheless present in each of Figs. $3 b-f$. These figures also have in common variability near 6 sols, although this feature is not very pronounced in Figs. 3c $(\mu=0.75), 3 \mathrm{e}(\mu=1.25)$, and $3 \mathrm{f}(\mu=1.5)$. This high-frequency variability is absent from the AAM 
(a)

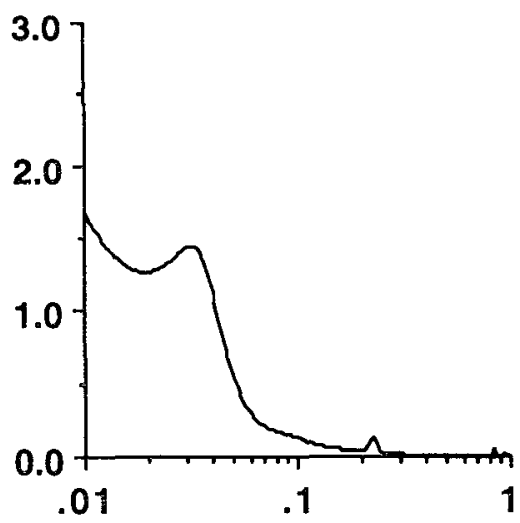

(d)

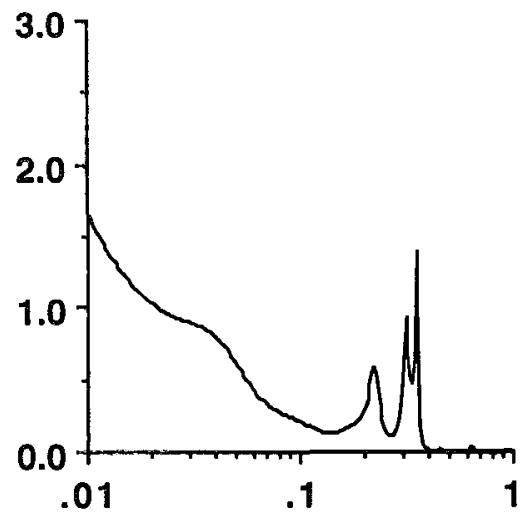

(b)

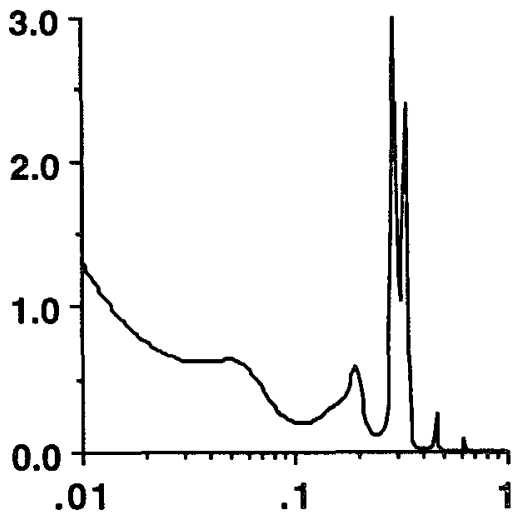

(e)

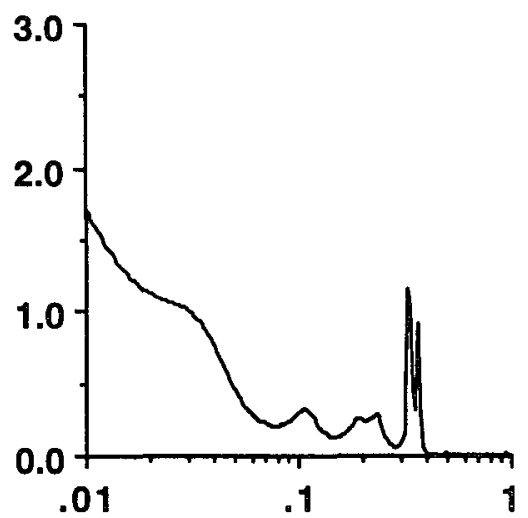

(c)

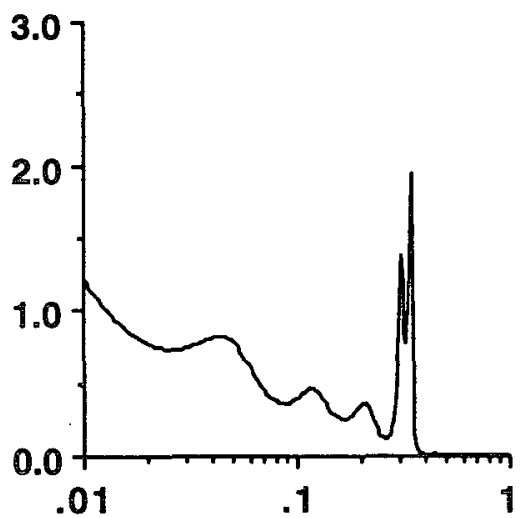

(g)

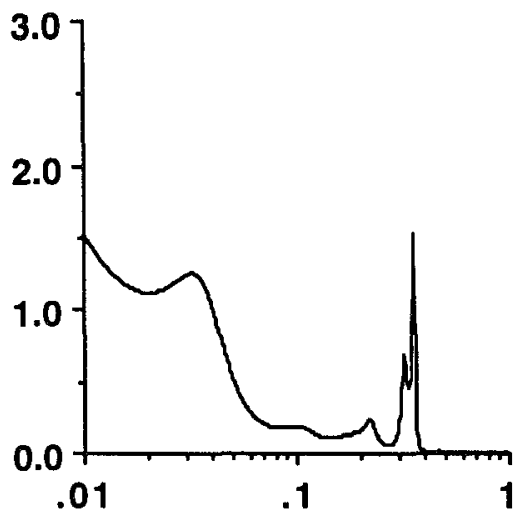

(h)

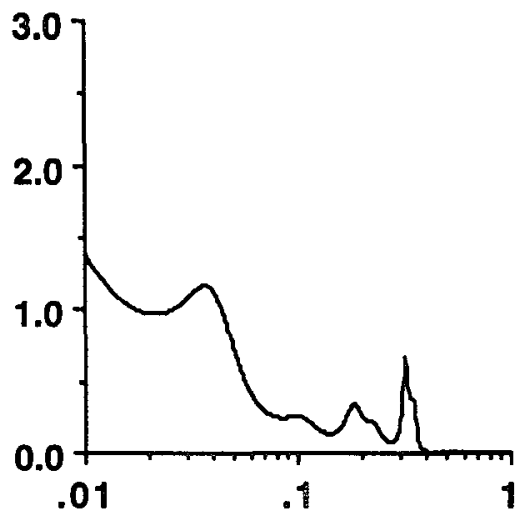

FIG. 3. Normalized power spectra of atmospheric angular momentum time series from 1000-sol model integrations with various parameter settings. (d) The nominal settings. (a) Corresponds to the run with no topography. The topography is multiplied by $0.5,0.75,1.25$, and 1.5 in the runs corresponding to (b), (c), (e), and (f), respectively. The relaxation parameter $\epsilon$ is half its nominal value in the run leading to ( $\mathrm{g}$ ) and twice that amount in that to which (h) corresponds. 
(a)

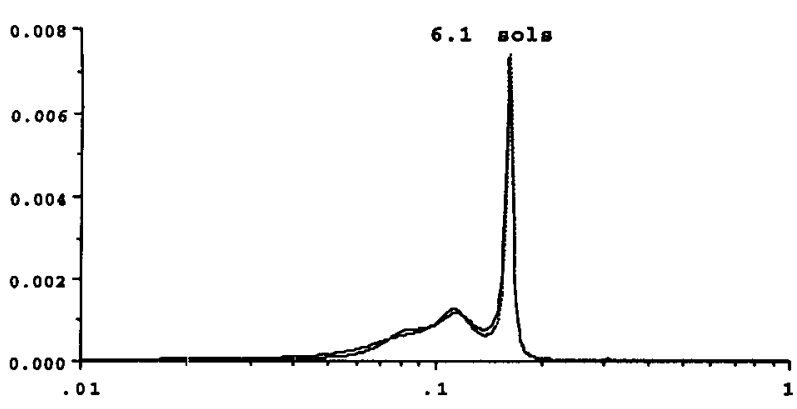

(b)

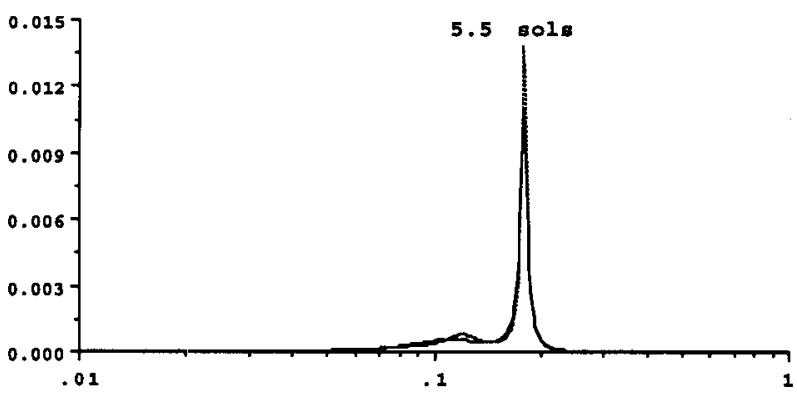

Fig. 4. Power spectra of (a) principal components (PCs) 3-4 and (b) PCs 8-9 resulting from the extended empirical orthogonal function (EEOF) decomposition of the geopotential history at the latitude $\left(48^{\circ} \mathrm{N}\right)$ of VL2. As in Fig. 2, the frequency axes are labeled in $\mathrm{sol}^{-1}$. The PSD is multiplied by frequency, and the vertical units are dimensionless because the PCs have been normalized to unity. The solid (dashed) lines show the power spectra corresponding to the lower-order (higher-order) PC of each pair.

power spectrum corresponding to the control run with no topography (Fig. 3a), thereby suggesting that it is orographically forced. The presence of the periodicities near 3 and 6 sols in all of Figs. 3b-f suggests that they are determined by the zonally asymmetric distribution of the mountains rather than by their height.

Figures $3 \mathrm{~g}$ and $3 \mathrm{~h}$ show AAM power spectra corresponding to first doubling (Fig. $3 \mathrm{~g}$ ) and then halving (Fig. $3 \mathrm{~h}$ ) the relaxation time, $1 / \epsilon$, in (1a). The periodicities near 3 and 6 sols are still present in both figures - thus, establishing their robustness with respect to changing the dissipation parameters - although the additional damping in the integration leading to Fig. $3 \mathrm{~h}$ noticeably affects the strength of the former.

Going back to the geopotential variability at the lander sites, the PSD associated with the 3-sol model oscillation at VL1 is about one order of magnitude less than that corresponding to the 6-sol oscillation at VL2. The same relationship exists between the PSDs associated with the observed 3.7- and 6.7-sol oscillations in the VL1 and VL2 pressure data (Barnes 1980). However, the power spectra of the model variables show much narrower peaks than those corresponding to the observations. The most probable explanation is that although the model may capture the part of the variability that is orographically forced during nearequinox conditions, it does not reproduce the dominant fraction that is induced by baroclinic instability. In the Martian atmosphere, the orographic forcing should reinforce the observed baroclinic oscillations. The 3- and 6-sol oscillations the model produces may contribute to the observed power found near the corresponding frequencies.

There are also some similarities between the model results and the Barnes (1980) observations in the phase lags between pressure and meridional velocity and in the meridional fluxes of zonal momentum and pressure: $v$ leads $\phi$ by about $80^{\circ}$ near VL2, in the model as in the observations, and-using primes to denote anomalies and angle brackets to indicate time averaging $\left\langle u^{\prime} v^{\prime}\right\rangle$ is positive at both lander sites. Also in agreement with the observations, $\left\langle v^{\prime} \phi^{\prime}\right\rangle$ is negative. Although there is little doubt that the observed phase lags and fluxes are mostly the reflection of a baroclinically unstable zonal jet, the results obtained by varying the height of the Martian topography indicate that the model oscillations are due to orographically forced barotropic waves.

To analyze the longitudinal character of the 3- and 6-sol orographically forced model oscillations identified at the lander sites, EEOF decomposition (e.g., Weare and Nasstrom 1982; Lau and Chan 1986; Graham et al. 1987a,b) is applied to multivariate time series of geopotential anomaly at the corresponding latitudes. EEOF analysis is a variant of principal component analysis (PCA: e.g., Preisendorfer 1988; Golub and Van Loan 1989) applied simultaneously in the temporal and spatial domains. The algorithm provides the basis vectors of a new coordinate system, the EEOFs, and the corresponding principal components (PCs), which give the representation of the model's trajectory in the new coordinate system. The main advantage of the EEOFs as basis vectors is that they are data-adaptive and indicate the directions of phase space along which the variability along the model's trajectory is maximum (e.g., Mo and Ghil 1987; Ghil 1988). The data-adaptive character of the method makes it possible to distinguish between oscillations of nearby periods whose signatures are blurred into a single peak in the power spectra of Fig. 2.

EEOF analysis is applied to the geopotential anomaly at the latitude of VL2 with a time window of 10 sols. The resulting singular spectrum contains two pairs of singular values (SVs) of nearly equal size. Such pairs have a particular significance in that they isolate an anharmonic oscillation from the remaining variability and noise (Vautard and Ghil 1989). The EEOFs associated with those pairs isolate $13.2 \%$ and $7.6 \%$ of the total variance, respectively. The MEM spectra of their respective PCs are shown in Fig. 4. The first pair (PCs 3-4) carries a 6.1-sol oscillation, while the sec- 
ond pair ( $\mathrm{PCs} 8-9$ ) oscillates with a period of 5.5 sols. The 6-sol variability observed in the model geopotential at VL2 can be understood as a combination of the two oscillations carried by the two pairs of EEOFs and PCs observed at this latitude.

Figure 5 shows time-longitude diagrams of EEOFs 3-4 and 8-9. Like the corresponding PCs, the two EEOFs of each pair are in quadrature, as can be expected from pairs of empirical orthogonal functions and PCs carrying the same oscillation (Vautard and Ghil 1989). Figures 5a-b show an eastward propagating mixed zonal wavenumber one and two pattern. The phase speed associated with this pattern is about 17 $\mathrm{m} \mathrm{s}^{-1}$. The mixed zonal wavenumber three and four pattern in Figs. 5c-d also propagates eastward, this time at about half the other pattern's speed. These phase speeds are in the broad range of phase speeds estimated by Barnes (1980) from the lander observations.

When EEOF analysis is applied with the same time window to the geopotential anomaly at the latitude of VL1, the method isolates one pair of singular values of nearly equal size. This pair (SVs 3-4) carries $9.2 \%$ of the variability at that latitude. The MEM spectra of the corresponding PCs, shown in Fig. 6, reveal a single peak at a frequency of $1 / 3.2 \mathrm{sol}^{-1}$. Figure 1 shows that this frequency dominates the variability east of VL1. Figure 7 shows the associated EEOFs. They isolate an eastward traveling zonal wavenumber one pattern. This pattern travels much faster than the midlatitude oscillations discussed in the previous paragraph. It revolves around the globe at $55 \mathrm{~m} \mathrm{~s}^{-1}$. Although these results are obtained with equinoctial wind forcing, the 55 $\mathrm{m} \mathrm{s}^{-1}$ phase speed is in agreement with Murphy et al.'s (a)

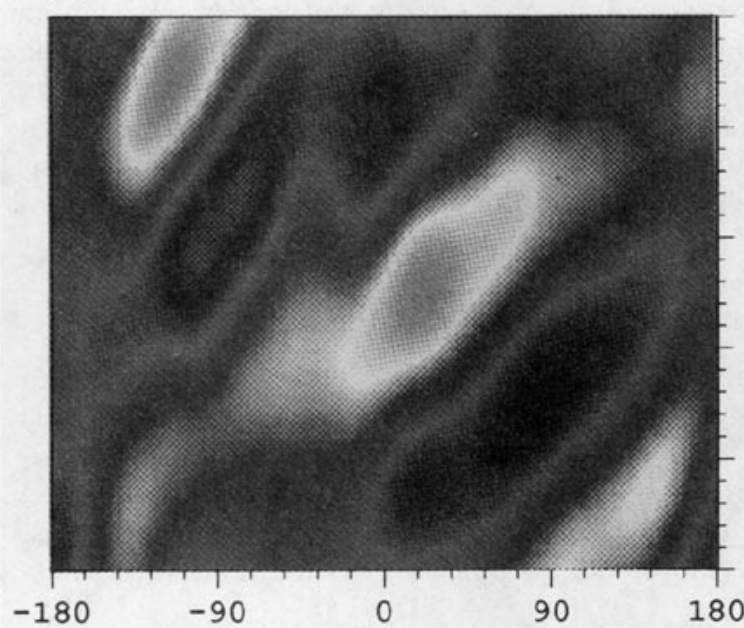

(c)

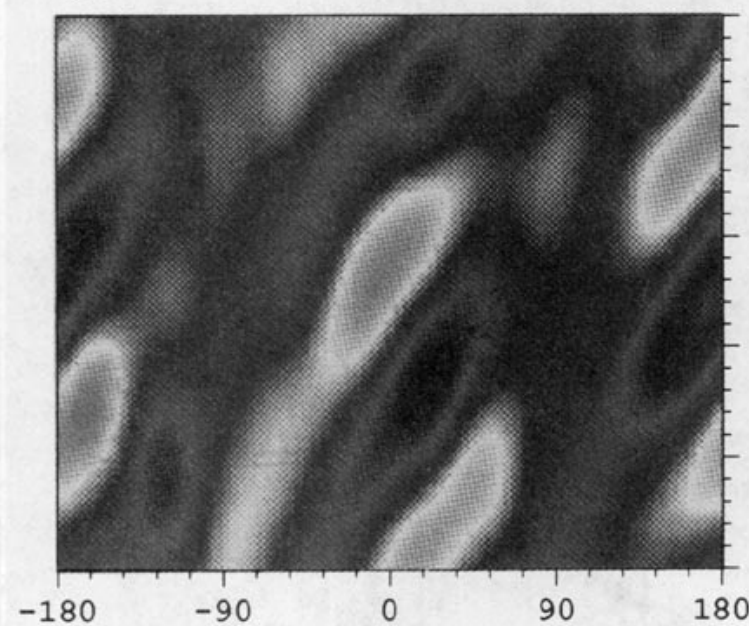

(b)

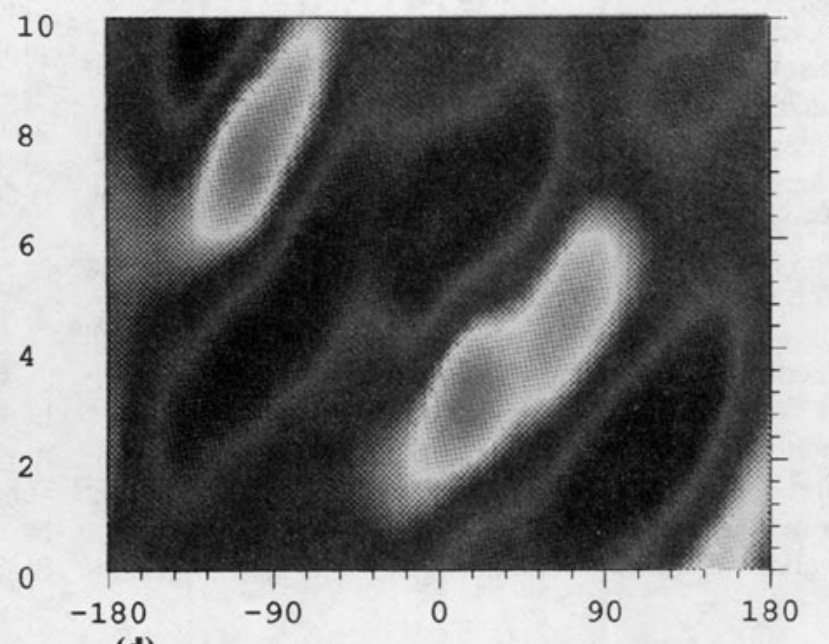

(d)

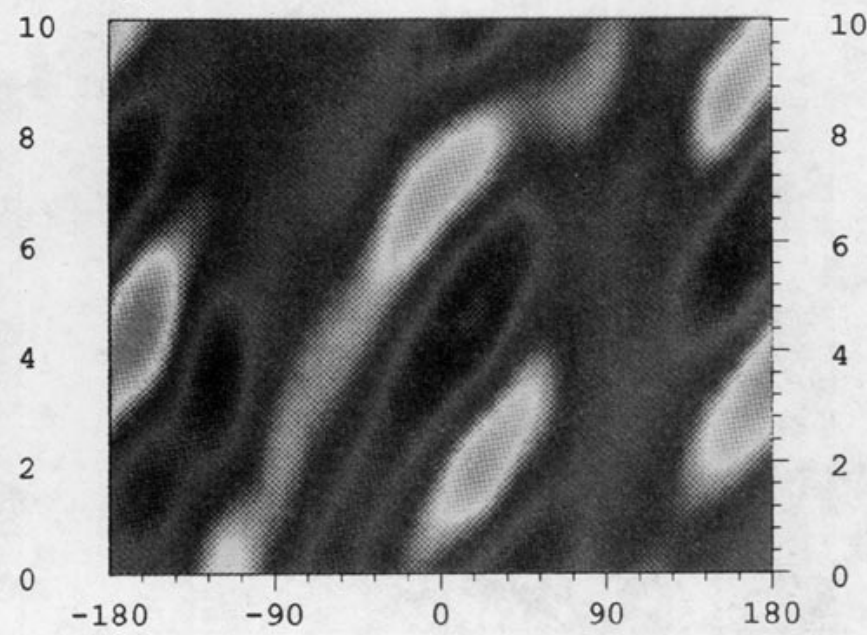

FIG. 5. Time-longitude diagrams of EEOFs (a)-(b) 3-4 and (c)-(d) 8-9 resulting from the EEOF decomposition of the geopotential history at the latitude of VL2. The horizontal and vertical axes, respectively, indicate the longitude in degrees and the time dimension of the EEOFs in sols. 


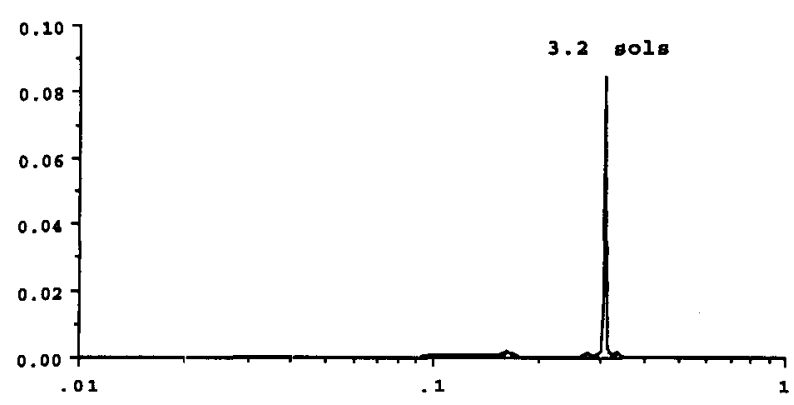

FIG. 6. Same as Fig. 4 for PCs 3-4 resulting from the EEOF decomposition of the geopotential history at the latitude of VLl.

(1990) estimate of the phase speed of a 3.3-sol wave during winter.

To investigate the mechanisms that lead to the strong periodicities identified in the model at VL1 and VL2, we linearize the model about its climatology and compute the eigenvalues and eigenvectors of the linear system. The model has 440 complex degrees of freedom for each of the $\psi, \chi$, and $\phi$ fields. Since the spectral coefficients associated with the zonal wavenumbers have zero imaginary parts, there are a total of 2460 real degrees of freedom. The Jacobian matrix considered in the analysis is that of the equivalent 2460-dimensional real system. The geopotential variable of the climatology is shown in Fig. 8b.

The largest growth rate is $0.47 \mathrm{sol}^{-1}$, corresponding to an $e$-folding time of about 2 sols. It is associated with complex conjugate eigenvalues $\left(0.47 \mathrm{sol}^{-1} \pm 0.18\right.$ $i \mathrm{sol}^{-1}$ ). The real part of the geopotential of the corresponding complex-conjugate eigenvectors is shown in Fig. 8c. It is dominated by a zonal wavenumber three pattern near the latitude of VL2 and by wavenumber two and wavenumber one patterns north thereof and in the high southern latitudes, respectively. The imaginary parts of the eigenvalues are indicative of a periodicity of $5.5 \mathrm{sols}$; close to the dominant periodicity in the model geopotential power spectrum at VL2 (Fig. 2b). The wavenumber-three dominance in the northern midlatitudes is also consistent with the results of the EEOF analysis at the latitude of VL2 $\left(48^{\circ} \mathrm{N}\right)$

The next-to-largest growth rate, $0.31 \mathrm{sol}^{-1}$, also corresponds to complex-conjugate pairs of eigenvalues and eigenvectors. The geopotential real part of these eigenvectors is shown in Fig. 8d. It is characterized by a zonal wavenumber one pattern that switches sign near the equator. The imaginary parts of the corresponding eigenvalues are $\pm 0.35 \mathrm{sol}^{-1}$. Thus, they indicate a periodicity near 2.8 sols, close to the 3.6 -sol period found in the model geopotential power spectrum at VL1 (Fig. $2 a)$. The zonal wavenumber one pattern agrees with the EEOF results at the latitude of VLl $\left(22^{\circ} \mathrm{N}\right)$.

The above results suggest that the orographically forced oscillations identified at VL1 and VL2 arise from two global instabilities of the model's zonally asymmetric climatology in the presence of the Martian mountain field. Strictly speaking, a linear analysis such as the one conducted here should be performed about a steady-state solution of the model rather than about its climatology. Nevertheless, the occurrence of positive eigenvalues in this analysis is indicative of a linearly unstable model climatology.

\section{Discussion}

The model results suggest that orographically forced barotropic oscillations with periods near 3 and 6 sols may play a role in determining the Martian atmosphere's $1-10$ sol variability by reinforcing observed baroclinically unstable modes with nearby periods. Since the model's single-layer configuration prevents (a)

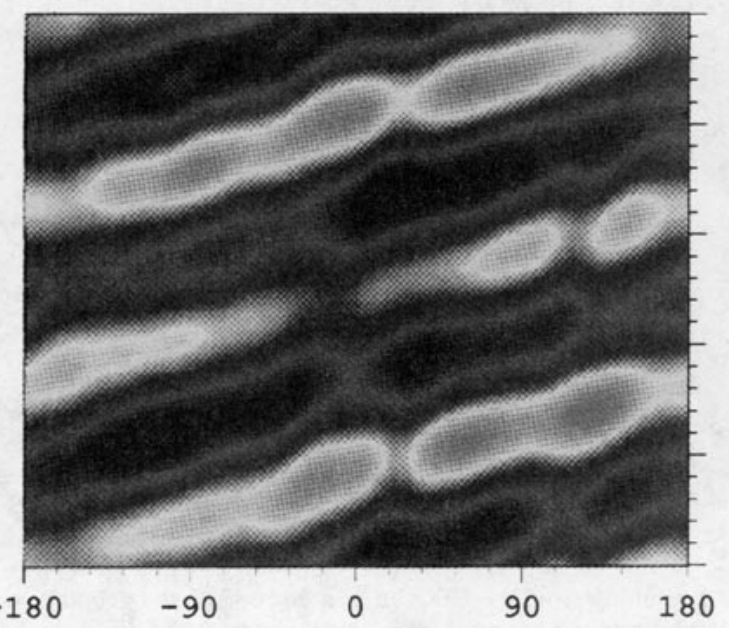

(b)

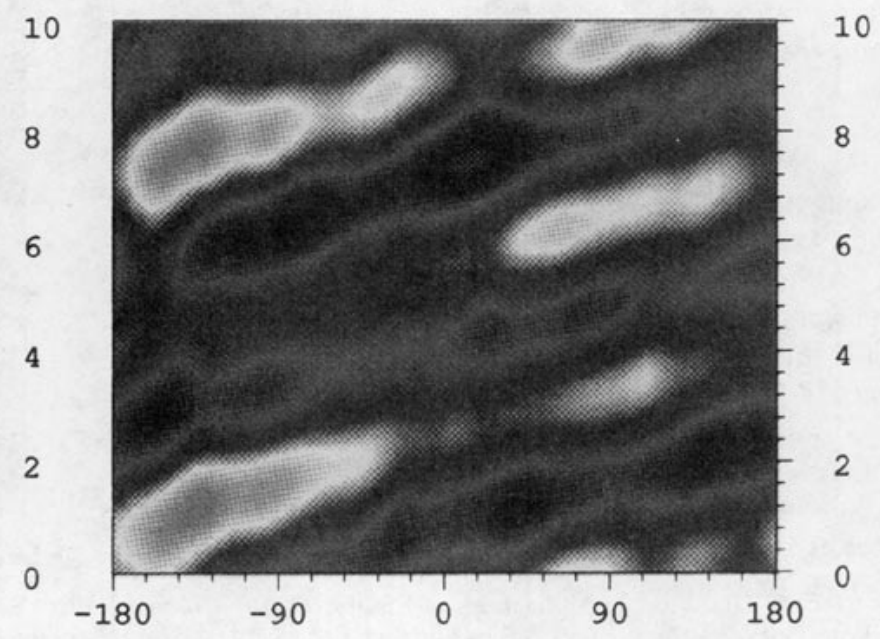

FIG. 7. Same as Fig. 5 for EEOFs (a)-(b) 3-4 resulting from the EEOF decomposition of the geopotential history at the latitude of VL1. 
(a)

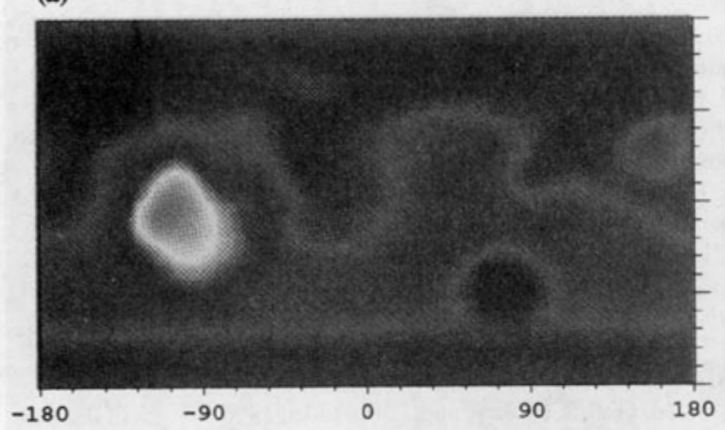

(c)

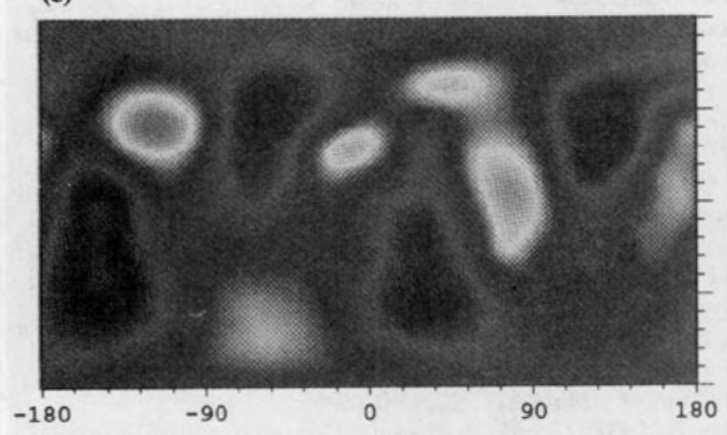

(b)

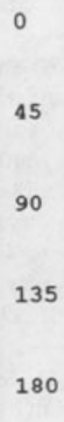

0
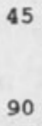

135

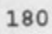

(d)
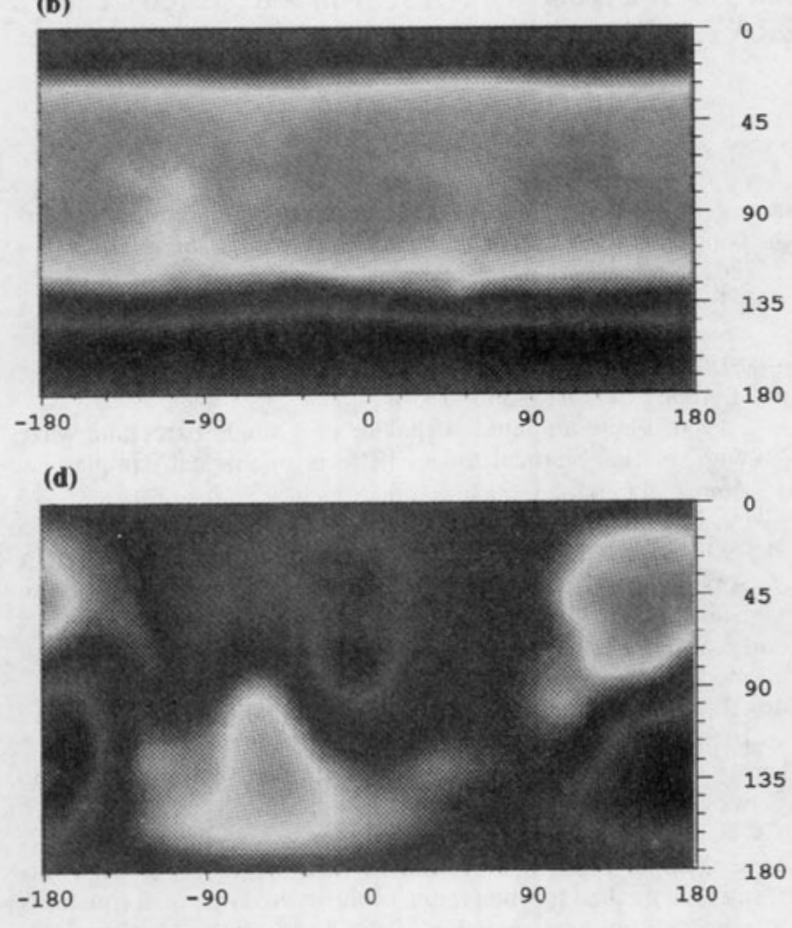

Fig. 8. Two-dimensional raster plots of (a) the model topography, (b) the time-mean geopotential field, and (c) and (d) the real part of the geopotential component of the two most unstable eigenvectors of the linearization of the model about its zonally asymmetric climatology. The color palette is shown on top of the figure. Values range from $-21.3 \mathrm{~km}$ to $35.4 \mathrm{~km}$ in (a) and from $19.2 \mathrm{~km}$ to $38.1 \mathrm{~km}$ in (b). Arbitrary units are used to display the eigenvectors shown in (c) and (d).

the manifestation of baroclinic waves, and since its response to the idealized equinoctial zonal forcing is barotropically stable if the longitudinal variability of the Martian mountain field is ignored, we conclude that the orographically forced modes are induced by the interaction of the barotropic zonal flow with this longitudinal variability. In the latter's presence, the model climatology is linearly unstable and the longitudinal structure of the eigenvectors associated with the two largest positive growth rates is reminiscent of the longitudinal variability identified in the model by means of EEOF analysis at the latitudes of VL1 and VL2. The periods associated with these growth rates are also close to those detected in the local model geopotential time series at the lander sites.

Although the model's orographically forced variance is comparable to the observed pressure variance, it is still unclear what fraction of the observed variability near 3 and 6 sols is caused by orographically forced barotropic oscillations such as those identified in the present study. The power spectra of Figs. 2, 3, and 5 suggest that this fraction may be fairly small, since their peaks are sharper, narrower, and involve less total power than those associated with nearby-period oscillations in the lander observations. A similar analysis with a multilayer version of the model would help an- swer this question by revealing how much more power is concentrated near these periods when baroclinic instability is allowed to occur. We feel that the most significant result of the present study is the manifestation of the orographically forced modes at periods within the broad range of those observed in the lander data. Since earlier studies have shown that the range of observed periods and zonal wavenumbers are baroclinically unstable (e.g., Barnes 1984, 1986), the orographically induced reinforcement of the near-3-sol and near6-sol instabilities should contribute to the excitement of these periods.

Acknowledgments. The authors acknowledge conversations with $\mathbb{D}$. J. Banfield, D. H. Boggs, J. O. Dickey, M. Ghil, and S. L. Marcus. They also thank Jim Pollack for providing the Martian GCM topography and three anonymous referees for their constructive criticisms and suggestions.

The research described in this publication was carried out in part by the Jet Propulsion Laboratory (JPL), California Institute of Technology (CIT), under a contract with the National Aeronautics and Space Administration, and in part by the Climate Dynamics Center, University of California, Los Angeles. It was funded through the JPL/CIT Director's Discretionary Fund for 
1992. The model development and simulations took place on JPL's and the NASA Ames Research Center's CRAY Y-MP computers.

\section{REFERENCES}

Barnes, J. R., 1980: Time spectral analysis of midlatitude disturbances in the Martian atmosphere. J. Atmos. Sci., 37, 20022015.

- 1981 : Midlatitude disturbances in the Martian atmosphere: A second Mars year, J. Atmos. Sci., 38, 225-234.

1984: Linear baroclinic instability in the Martian atmosphere. J. Atmos. Sci., 41, 1536-1550.

_ 1 1986: Finite amplitude behavior of a single baroclinic wave with multiple vertical modes: Effects of thermal damping. $J$. Atmos. Sci., 43, 58-71.

- J. B. Pollack, R. M. Haberle, C. B. Leovy, and R. W. Zurek, 1993: Mars atmospheric dynamics as simulated by the NASA Ames general circulation model. Part 2: Transient baroclinic eddies. J. Geophys. Res., 98, 3125-3148.

Bourke, W., 1972: An efficient, one-level, primitive-equation spectral model. Mon. Wea. Rev., 100, 683-689.

Burg, J., 1968: Maximum entropy spectral analysis. Modern Spectrum Analysis, D. G. Childers, Ed., IEEE Press, 34-48.

Charney, J. G., 1947: The dynamics of long-waves in a baroclinic westerly current. J. Meteor., 4, 135-162.

Eady, E. T., 1949: Long waves and cyclone waves. Tellus, 1, 33-52.

Eliassen, E., B. Machenhauer, and E. Rassmusen, 1970: On a numerical method for integration of the hydrodynamical equations with spectral representation of the horizontal fields. Report 2 , Institute for Theoretical Meteorology, University of Copenhagen, $35 \mathrm{pp}$

Ghil, M., 1987: Dynamics, statistics and predictability of planetary flow regimes. Irreversible Phenomena and Dynamical Systems Analysis in Geosciences, C. and G. Nicolis, Eds., Reidel, 241283.

- 1988: Nonlinear approaches to low-frequency atmospheric variability. Dynamics of Low-Frequency Phenomena in the Atmosphere, G. Branstator et al., Eds., National Center for Atmospheric Research, 603-714.

Golub, G. H., and C. F. Van Loan, 1989: Matrix Computations. The Johns Hopkins University Press, 642 pp

Graham, N. E., J. Michaelsen, and T. P. Barnett, 1987a: An investigation of the El Niño-STouthern Oscillation cycle with statistical models. Part 1: Predictor field characteristics. J. Geophys. Res. 92, $14251-14270$.

,-- , and,$- 1987 \mathrm{~b}$ : An investigation of the El NiñoSouthern Oscillation cycle with statistical models. Part 2: Model results. J. Geophys. Res., 92, 14 271-14 289.

Haberle, R. M., J. B. Pollack, J. R. Barnes, R. W. Zurek, C. B. Leovy, J. R. Murphy, H. Lee, and J. Schaeffer, 1993: Mars atmospheric dynamics as simulated by the NASA Ames general circulation model 1. The zonal mean circulation. J. Geophys. Res., 98, 3093-3123.

Jin, F-f., and M. Ghil, 1990: Intraseasonal oscillations in the extratropics: Hopf bifurcation and topographic instabilities. J. Atmos. Sci., 47, 823-839.
Keppenne, C. L., 1992: Orographically forced oscillations in a dynamical model of the Martian atmosphere. Icarus, 100, 598607

— - and M. Ghil, 1995: Intraseasonal oscillations in a two-layer model. J. Atmos. Sci., 52, submitted.

Lau, K. M., and P. H. Chan, 1986: Aspects of the 40-50 day oscillation during the northern summer as inferred from outgoing longwave radiation. Mon. Wea. Rev., 114, 1354-1367.

Legras, B., and M. Ghil, 1985: Persistent anomalies, blocking and variations in atmospheric predictability. J. Atmos. Sci., 43, 433471.

Leovy, C. B., 1969: Mars: Theoretical aspects of meteorology. Appl. Opt., 8, 1279-1286.

—, 1979: Martian meteorology. Ann. Rev. Astron. Astrophys., 17, $387-413$.

Marcus, S., M. Ghil, J. Dickey, and T. Eubanks, 1990: Origin of the 30 60 day oscillation in the length of day and atmospheric angular momentum: New findings from the UCLA general circulation model. Earth Rotation and Reference Frames for Geodesy and Geodynamics, G. A. Wilkins, Ed., Springer-Verlag, 98-105.

Mintz, Y., 1961: The general circulation of planetary atmospheres. The Atmospheres of Mars and Venus, NAS-NRC, Publ. 944, $107-146$.

Mo, K. C., and M. Ghil, 1987: Statistics and dynamics of persistent anomalies. J. Atmos. Sci., 44, 877-901.

Murphy, J. R., C. B. Leovy, and J. E. Tillman, 1990: Observations of Martian surface winds at the Viking Lander 1 site. J. Geophys. Res., 95(B9), 14 555-14 576

Orszag, S., 1970: Transform method for the calculation of vectorcoupled sums: Application to the spectral form of the vorticity equation. J. Atmos. Sci., 27, 890-895.

Pedlosky, J., 1987: Geophysical Fluid Dynamics. 2d ed. SpringerVerlag, $710 \mathrm{pp}$.

Pollack, J., C. Leovy, P. Greiman, and Y. Mintz, 1981: A Martian general circulation experiment with large topography. J. Atmos. Sci., 38, 3-29.

— R. Haberle, J. Schaeffer, and H. Lee, 1990: Simulation of the general circulation of the Martian atmosphere. Part I: Polar processes. J. Geophys. Res., 95, 1447-1474.

Preisendorfer, R. W., 1988: Principal Component Analysis in Meteorology and Oceanography. Elsevier, $425 \mathrm{pp}$.

Strong, C., F-f. Jin, and M. Ghil, 1993: Intraseasonal oscillations and the stability of a seasonally-forced barotropic model. Preprints, Ninth Conf. on Atmospheric and Oceanic Waves and Stability, San Antonio, TX, Amer. Meteor. Soc., 389-392.

Tribbia, J., and M. Ghil, 1990: Forced zonal flow over topography and the 30-60 day oscillation in atmospheric angular momentum. NCAR 0501/89-5, National Center for Atmospheric Research, Boulder, CO, 26 pp.

Vautard, R., and M. Ghil, 1989: Singular spectrum analysis in nonlinear dynamics, with applications to paleoclimatic time series. Physica D, 35, 395-424.

Walker, G., 1931: On periodicity in series of related terms. Proc. Roy. Soc. London Series A, 131, 518-532.

Weare, B. C., and J. S. Nasstrom, 1982: Examples of extended empirical orthogonal function analyses. Mon. Wea. Rev., 110, 481485.

Yule, G., 1927: On a method of investigating periodicities in disturbed series. Philos. Trans. Roy. Soc. London Series A, 226, 267-298. 